\title{
Differences of tumor microenvironment between stage I lepidic-positive and lepidic-negative lung adenocarcinomas
}

\author{
Shinya Katsumata, MD, ${ }^{\mathrm{a}, \mathrm{b}, \mathrm{c}}$ Keiju Aokage, MD, PhD, ${ }^{\mathrm{b}}$ Tomohiro Miyoshi, MD, PhD, ${ }^{\mathrm{b}}$ Kenta Tane, MD, ${ }^{\mathrm{b}}$ \\ Hiroshi Nakamura, MD, ${ }^{\text {add }}$ Masato Sugano, MD, ${ }^{\mathrm{d}}$ Motohiro Kojima, MD, PhD, ${ }^{\mathrm{a}}$ Satoshi Fujii, MD, PhD, ${ }^{\mathrm{a}}$ \\ Takeshi Kuwata, MD, PhD, ${ }^{\mathrm{d}}$ Atsushi Ochiai, MD, PhD, ${ }^{\mathrm{e}}$ Ryuichi Hayashi, MD, ${ }^{\mathrm{c}, \mathrm{f}}$ \\ Masahiro Tsuboi, MD, PhD, ${ }^{b}$ and Genichiro Ishii, $\mathrm{MD}, \mathrm{PhD}^{\mathrm{a}}$
}

\section{ABSTRACT}

Objective: Lepidic growth is a noninvasive component of lung adenocarcinoma. Many adenocarcinoma cases contain coexistent lepidic and nonlepidic (invasive) components (lepidic-growth positive [Lep+] adenocarcinoma); however, some cases comprise only nonlepidic components (lepidic-growth negative [Lep-] adenocarcinoma). The aim of this study was to investigate the biological differences between the invasive components of Lep + and Lep- adenocarcinoma.

Methods: We investigated the clinicopathologic characteristics of 232 adenocarcinomas (116 size-matched tumor pairs from Lep+ and Lep- adenocarcinomas). We then evaluated the cancer cell-specific expression levels of cancer stem cell, hypoxia, and invasion molecules in these lesions. The number of tumorpromoting stromal cells, including podoplanin-positive cancer-associated fibroblasts and CD204-positive tumor-associated macrophages, was also analyzed.

Results: Among cases with size-matched invasive components, significant differences were shown in total tumor size and predominant subtype in invasive component between Lep + and Lep- adenocarcinomas. The expression levels of hypoxia-related molecules were significantly lower in Lep + adenocarcinomas (glucose transporter 1: 0 vs $10, P<.01$; carbonic anhydrase IX: 0 vs 0 [mean, 4.7 vs 14.1$], P=.01)$. The number of podoplanin-positive cancer-associated fibroblasts and CD204-positive tumor-associated macrophages was significantly lower in Lep + adenocarcinomas (podoplanin-positive cancer-associated fibroblasts: 0 vs 0 [mean: 1.6 vs 11.6], $P<.01$; CD204-positive tumor-associated macrophages: 8.7 vs $24.7, P<.01)$.

Conclusions: Our results indicated that lower cancer cell-specific expression levels of hypoxia markers and a smaller number of tumor-promoting stromal cells in invasive component were characteristic features of Lep+ adenocarcinomas. (J Thorac Cardiovasc Surg 2018;156:1679-88)

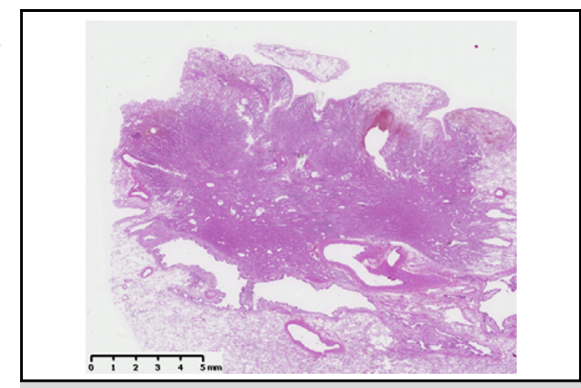

Lep+ adenocarcinomas are less malignant than invasive size-matched Lep- adenocarcinomas.

\section{Central Message}

Lower cancer cell-specific expression levels of hypoxia markers and a smaller number of tumor-promoting stromal cells in invasive component were characteristic features of Lep+ lung adenocarcinomas.

\section{Perspective}

We characterized the clinicopathologic differences of the invasive components between Lep + and Lep- invasive size-matched tumors belonging in pathologic stage I. Given that Lep + adenocarcinoma is less invasive with less metastatic potential, early-stage small Lep+ adenocarcinomas may be treated with limited resection techniques such as widewedge resection and segmentectomy.

See Editorial Commentary page 1689.

See Editorial page 1669.

\footnotetext{
From the ${ }^{a}$ Division of Pathology, Exploratory Oncology Research \& Clinical Trial Center, National Cancer Center, Kashiwa, Chiba, Japan; ${ }^{\mathrm{b}}$ Department of Thoracic Surgery, National Cancer Center Hospital East, Kashiwa, Chiba, Japan; ${ }^{\mathrm{c} C o u r s e}$ of Advanced Clinical Research of Cancer, Juntendo University Graduate School of Medicine, Tokyo, Japan; ${ }^{\mathrm{d}}$ Department of Pathology and Clinical Laboratories, National Cancer Center Hospital East, Kashiwa, Chiba, Japan; ${ }^{e}$ Exploratory Oncology Research and Clinical Trial Center, National Cancer Center, Kashiwa, Chiba, Japan; and ${ }^{\mathrm{f}}$ Department of Head and Neck Surgery, National Cancer Center Hospital East, Kashiwa, Chiba, Japan.
}

Funding: This work was supported in part by the National Cancer Center Research and Development Fund (23-A-12, 28-seeds-2), the Foundation for the Promotion of Cancer Research, and JSPS KAKENHI (24659185 and 16H05311).

Received for publication Dec 16, 2017; revisions received April 27, 2018; accepted for publication May 17, 2018; available ahead of print June 29, 2018.

Address for reprints: Genichiro Ishii, MD, PhD, Division of Pathology, Exploratory Oncology Research and Clinical Trial Center, 6-5-1 Kashiwanoha, Kashiwa, Chiba 277-8577, Japan (E-mail: gishii@east.ncc.go.jp).

$0022-5223 / \$ 36.00$

Copyright (C) 2018 by The American Association for Thoracic Surgery

https://doi.org/10.1016/j.jtcvs.2018.05.053 


$$
\begin{aligned}
& \text { Abbreviations and Acronyms } \\
& \begin{aligned}
\text { CA } & =\text { carbonic anhydrase } \\
\text { CAF } & =\text { cancer-associated fibroblast } \\
\text { CT } & =\text { computed tomography } \\
\text { GGO } & =\text { ground-glass opacity } \\
\text { GLUT-1 } & =\text { glucose transporter } 1 \\
\text { HE } & =\text { hematoxylin-eosin } \\
\text { IHC } & =\text { immunohistochemical } \\
\text { Lep+ } & =\text { lepidic-growth positive } \\
\text { Lep- } & =\text { lepidic-growth negative } \\
\text { PBS } & =\text { phosphate-buffered saline } \\
\text { PDPN } & =\text { podoplanin } \\
\text { TAM } & =\text { tumor-associated macrophage }
\end{aligned}
\end{aligned}
$$

\section{Scanning this $Q R$ code will take you to supplemental figures and tables for this article.}

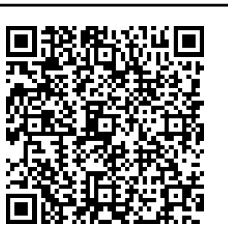

Lung cancer is one of the leading global causes of mortality. Adenocarcinoma, the most common histologic subtype of this disease, ${ }^{1}$ is heterogeneous with respect to its molecular biology; clinical, pathologic, and radiologic features; and surgical approach. Adenocarcinoma is primarily classified as 1 of 5 histopathologic subtypes, including lepidic, papillary, acinar, solid, and micropapillary. It is widely accepted that adenocarcinoma cells exhibiting a lepidic growth pattern are noninvasive tumor components. ${ }^{2,3}$ Therefore, the prognostic importance of invasive component size, excluding the lepidic component, has gained gradual recognition. ${ }^{4}$ Indeed, the TNM classification was drastically revised in 2016, especially in T category, and the size of the invasive component is now predominantly used to determine the T category. ${ }^{2}$ Although the Eighth TNM classification reflects the prognosis of patients with lung cancer, many patients with stage I disease still have unfavorable prognoses. ${ }^{5}$ This discrepancy indicates that factors other than invasive component size may influence prognosis.

The biological and morphologic characteristics of the invasive component are important determinants of cancer cell malignant potential. ${ }^{6,7}$ In addition to cancer cells, the invasive component comprises several stromal cell types, including macrophages, inflammatory cells, and fibroblasts. These stromal cells can interact with cancer cells and create a specific tumor microenvironment, influencing cancer cell proliferation, invasion, and metastasis. Given the influence of stromal cells on clinical tumor features, characterization of stromal cell phenotypes may improve our understanding of patient prognosis. ${ }^{8-11}$

Typical adenocarcinoma cases contain both lepidic and nonlepidic (invasive) components (lepidic-growth positive [Lep+] adenocarcinomas). Computed tomography (CT) findings of these cases often include both ground-glass opacity (GGO) in the periphery and a solid component in the center. Conversely, some purely invasive cases, such as papillary, acinar, and solid adenocarcinoma (lepidicgrowth negative [Lep-] adenocarcinoma), typically only exhibit a solid component on CT. In clinical investigations, the presence of a GGO component may indicate a good prognosis in patients with an early clinical stage. ${ }^{12}$ Of note, Moon and colleagues ${ }^{13}$ reported that the lack of a lepidic component was associated with an unfavorable prognosis, even in patients with stage I. As a result, the presence of a lepidic GGO pattern in lung adenocarcinoma may confer a favorable prognosis.

We hypothesized that the tumor microenvironment of the invasive component of Lep+ adenocarcinoma would differ from that of Lep- adenocarcinoma and that this relationship would persist in lesions with size-matched invasive (nonlepidic) components. To this end, we characterized the clinicopathologic differences between the invasive components of Lep+ and Lep- invasive size-matched tumors.

\section{MATERIALS AND METHODS Patients}

A retrospective review of data from accumulated the Thoracic Surgical Database in National Cancer Center Hospital East was performed. Patients who underwent R0 resection by lobectomy or more for lung adenocarcinoma at our institution from January 2003 to December 2011 were enrolled in this study. We excluded patients who underwent preoperative treatment and patients with insufficient data. We classified them according to the pathologic status defined by the 2004 World Health Organization classification and the Eighth edition of the TNM classification of the Union for International Cancer Control. Stage IA adenocarcinomas measuring $3 \mathrm{~cm}$ or less $(n=575)$ were then divided into 2 groups: Lep + adenocarcinomas (lepidic component ratio in their tumor of $\geq 10 \%$ ) and Lep- adenocarcinomas (lepidic component ratio of $<10 \%$ ) (Table 1 and Figure 1).

All studies involving human participants were performed in accordance with the ethical standards of the institutional and national research committees, and with the 1964 Declaration of Helsinki, its later amendments, or comparable ethical standards. Comprehensive informed consent was obtained in the study.

\section{Histopathologic Evaluation}

Resected specimens were fixed with $10 \%$ formalin and embedded in paraffin, and $4-\mu \mathrm{m}$ sections were stained with hematoxylin-eosin (HE) stain. Vascular and lymphovascular invasion were identified by HE and the Victoria blue-van Gieson, visualizing elastic fibers, staining. Histologic subtype and total and invasive size were reviewed by S.K. and G.I., and tumors were classified according to the 2015 World Health Organization Classification of Tumors of the Lung, Pleura, Thymus, and Heart. Tumor staging was based on the Eighth Edition of the TNM classification of the International Association for the Study of Lung Cancer. 


\section{Matching of Invasive Size}

We matched the invasive component sizes of selected tumors (optimal matching with no caliper) statistically using EZR (Saitama Medical Center, Jichi Medical University; see "Statistical Methods"). Patients were allocated in a 1:1 ratio, and 2 groups with the same number of patients ( $\mathrm{n}=116$ per group) were selected for further analysis (Table 2). Standardized differences of both pre- and postmatched cohort are shown in Tables 1 and 2 .

\section{Immunohistochemical Staining}

Because we predicted that the biological differences between Lep $(+)$ and Lep(-) adenocarcinoma would be found even in early-stage cancer, we selected 62 pathologic T1b tumors from each group of matched cases for immunohistochemical (IHC) staining. One case with variant type (showing only intrabronchial growth) in Lep- adenocarcinomas was excluded ( $n=62$ Lep+ adenocarcinomas; $n=61$ Lep-adenocarcinomas). Slides were deparaffinized with xylene and dehydrated with a graded ethanol series. Endogenous peroxidase was blocked with 3\% hydrogen peroxide in absolute methyl alcohol. After epitope retrieval, slides were washed with phosphate-buffered saline (PBS) and incubated overnight at $4^{\circ} \mathrm{C}$ with primary antibodies at their final dilution in the blocking buffer. The primary antibodies we used are shown in Table E1. We chose cancer stem cell-related molecule (ALDH-1, CD44) because a higher expression level of cancer stem cell-related molecules was reported to be a prognostic marker and associated with a subtype of adenocarcinoma. To investigate the potential of invasiveness, proliferation, or hypoxic condition in cancer cells, we selected invasion-related molecule (laminin-5) and hypoxiarelated molecules (glucose transporter 1 [GLUT1], carbonic anhydrase [CA] IX). To research the tumor promoting stromal cells, we investigated PDPN + cancer-associated fibroblast (CAFs) and CD204+ tumorassociated macrophages (TAMs). ${ }^{11,14,15}$ Then after washing them with PBS again, they were incubated with EnVision (Agilent Technologies, Santa Clara, Calif) for 1 hour at room temperature. After washing them with PBS, the color reaction was performed with diaminobenzidine. Finally, these slides were counterstained with Meyer's hematoxylin, dehydrated, and mounted.

TABLE 1. Clinicopathologic characteristics of patient with pathologic stage IA adenocarcinoma

\begin{tabular}{|c|c|c|c|c|c|}
\hline Variables & $\frac{\text { Lepidic }(+)}{\text { No. }(\%)}$ & $\frac{\text { Lepidic (-) }}{\text { No. }(\%)}$ & Odds $(95 \% \mathrm{CI})$ & $\boldsymbol{P}$ & Standardized difference \\
\hline Overall & 459 & 116 & & & \\
\hline $\begin{array}{l}\text { Age }^{*} \\
\qquad 665 \mathrm{y} \\
\geq 65 \mathrm{y}\end{array}$ & $\begin{array}{l}200(44) \\
259(56)\end{array}$ & $\begin{array}{l}51(44) \\
65(56)\end{array}$ & $1.27(0.75-2.18)$ & .38 & 0.008 \\
\hline $\begin{array}{l}\text { Sex* } \\
\quad \text { Female } \\
\text { Male }\end{array}$ & $\begin{array}{l}268(58) \\
191(42)\end{array}$ & $\begin{array}{l}44(38) \\
72(62)\end{array}$ & $1.30(0.64-2.64)$ & .46 & 0.42 \\
\hline $\begin{array}{l}\text { Smoking* } \\
\text { Never } \\
\text { Ever }\end{array}$ & $\begin{array}{l}260(57) \\
199(43)\end{array}$ & $\begin{array}{l}34(29) \\
82(71)\end{array}$ & $2.25(1.06-4.75)$ & .03 & 0.57 \\
\hline $\begin{array}{l}\text { CEA (except } 1 \text { patient with data missing)* } \\
\quad \leq 5.0 \\
>5.0\end{array}$ & $\begin{array}{r}394(86) \\
64(14)\end{array}$ & $\begin{array}{l}87(75) \\
29(25)\end{array}$ & $1.46(0.73-2.93)$ & .28 & 0.28 \\
\hline $\begin{array}{l}\text { Total tumor size }(\mathrm{mm})^{*} \\
\text { Median }(\mathrm{IQR})\end{array}$ & $20(9.5)$ & $20(9.0)$ & & .09 & 0.18 \\
\hline $\begin{array}{l}\text { Invasive component size }(\mathrm{mm})^{*} \\
\quad \text { Median (IQR) }\end{array}$ & $10.5(10.8)$ & $19.5(9.0)$ & & $<.01$ & 1.27 \\
\hline $\begin{array}{l}\text { Pathologic T stage } \\
\text { T1mi } \\
\text { T1a } \\
\text { T1b } \\
\text { T1c }\end{array}$ & $\begin{array}{r}91(20) \\
135(29) \\
180(39) \\
53(12)\end{array}$ & $\begin{array}{c}0(0) \\
8(7) \\
62(53) \\
46(40)\end{array}$ & & $<.01$ & \\
\hline $\begin{array}{l}\text { Lymphatic invasion* } \\
\text { Absent } \\
\text { Present }\end{array}$ & $\begin{array}{c}444(97) \\
15(3)\end{array}$ & $\begin{array}{c}105(91) \\
11(9)\end{array}$ & $2.34(0.69-7.94)$ & .17 & 0.26 \\
\hline $\begin{array}{l}\text { Vascular invasion* } \\
\text { Absent } \\
\text { Present }\end{array}$ & $\begin{array}{c}419(91) \\
40(9)\end{array}$ & $\begin{array}{l}75(65) \\
41(35)\end{array}$ & $1.56(0.72-3.40)$ & .26 & 0.68 \\
\hline $\begin{array}{l}\text { EGFR mutation } \\
\text { Absent } \\
\text { Present } \\
\text { Not examined }\end{array}$ & $\begin{array}{c}15(3) \\
80(17) \\
364(79)\end{array}$ & $\begin{array}{l}14(12) \\
15(13) \\
87(75)\end{array}$ & & $<.01$ & \\
\hline
\end{tabular}

$C I$, Confidence interval; $C E A$, carcinoembryonic antigen; $I Q R$, interquartile range; $E G F R$, epidermal growth factor receptor. *Included in the logistic regression model. 


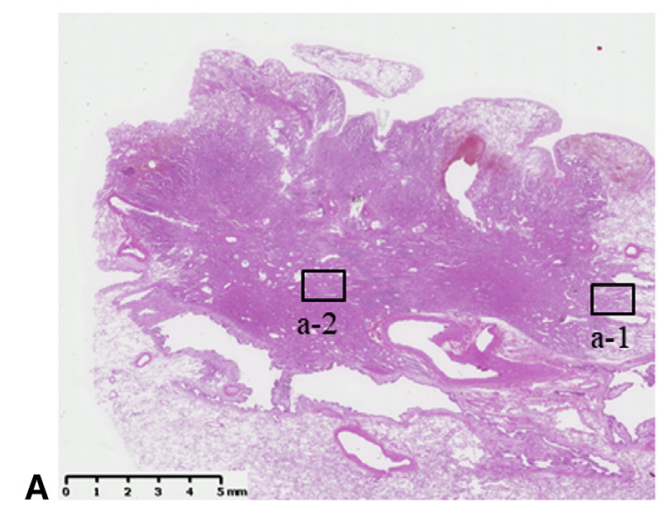

a-1

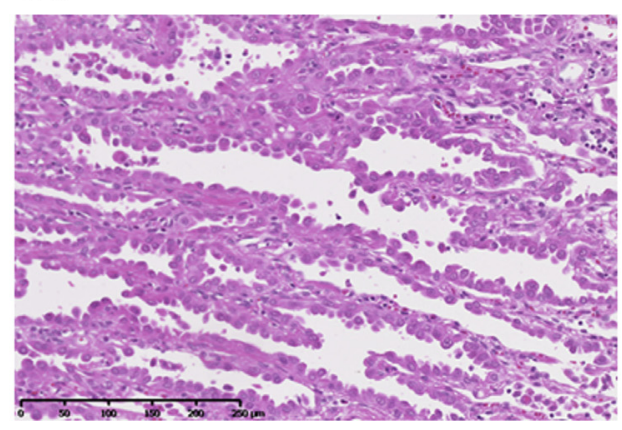

a-2

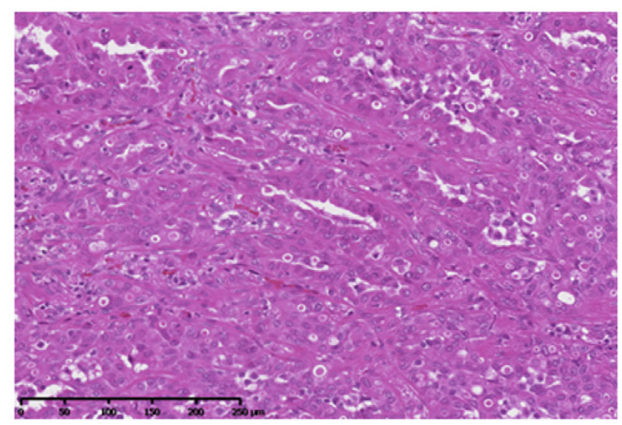

b-1

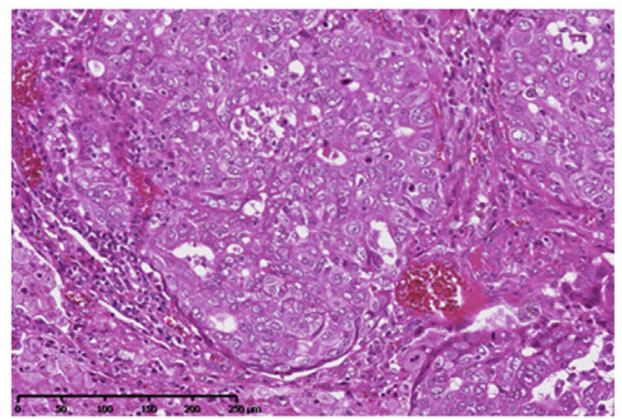

b-2

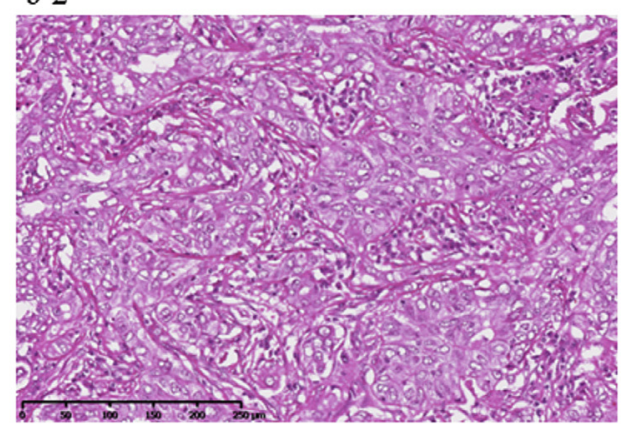

FIGURE 1. Microscopic features of Lep+ and Lep- adenocarcinomas. A, Low-power view of Lep+ adenocarcinomas. B, Low-power view of Lepadenocarcinomas. a: High-power view of Lep+ adenocarcinomas; 1: peripheral region, 2: central region. b: High-power view of Lep- adenocarcinomas; 1: peripheral region, 2: central region.

\section{Immunohistochemical Scoring Method}

Two pathologists (S.K. and G.I.) independently evaluated all tissue sections using light microscopy. Noninvasive and invasive component areas were determined using HE or Victoria blue-van Gieson-stained sections. The IHC staining scores of ALDH1, CD44, CA IX, GLUT-1, and laminin-
5 were evaluated on the basis of the staining intensity and the percentage, corresponding to every 10 percentages $(0 \%-100 \%)$, of stained cancer cells. We used the following scoring system: 0 (negative staining, defined as no immunoreactivity); $1+$ (weak staining intensity); $2+$ (strong staining intensity). We calculated staining scores by multiplying the stained percentages by the 
TABLE 2. Clinicopathologic characteristics of patient with pathologic stage IA adenocarcinoma after matching for invasive component size

\begin{tabular}{|c|c|c|c|c|c|}
\hline Variables & $\frac{\text { Lepidic }(+)}{\text { No. }(\%)}$ & $\frac{\text { Lepidic }(-)}{\text { No. }(\%)}$ & Odds $(95 \%$ CI $)$ & $P$ & Standardized difference \\
\hline Overall & 116 & 116 & & & \\
\hline $\begin{array}{l}\text { Age }^{*} \\
\qquad 665 \mathrm{y} \\
\geq 65 \mathrm{y}\end{array}$ & $\begin{array}{l}55(47) \\
61(53)\end{array}$ & $\begin{array}{l}51(44) \\
65(56)\end{array}$ & $1.38(0.40-4.79)$ & .61 & 0.07 \\
\hline $\begin{array}{l}\text { Sex* } \\
\quad \text { Female } \\
\text { Male }\end{array}$ & $\begin{array}{l}59(51) \\
57(49)\end{array}$ & $\begin{array}{l}44(38) \\
72(62)\end{array}$ & $0.24(0.03-2.06)$ & .19 & 0.26 \\
\hline $\begin{array}{l}\text { Smoking* } \\
\text { Never } \\
\text { Ever }\end{array}$ & $\begin{array}{l}53(46) \\
63(54)\end{array}$ & $\begin{array}{l}34(29) \\
82(71)\end{array}$ & $0.20(0.01-3.77)$ & .28 & 0.34 \\
\hline $\begin{array}{l}\text { CEA* } \\
\quad \leq 5.0 \\
>5.0\end{array}$ & $\begin{array}{l}95(82) \\
21(18)\end{array}$ & $\begin{array}{l}87(75) \\
29(25)\end{array}$ & $3.26(0.26-40.4)$ & .36 & 0.17 \\
\hline $\begin{array}{l}\text { Total tumor size }(\mathrm{mm})^{*} \\
\quad \text { Median }(\mathrm{IQR})\end{array}$ & $25(7.0)$ & $20(9.0)$ & & $<.01$ & 0.76 \\
\hline $\begin{array}{l}\text { Invasive component size }(\mathrm{mm})^{*} \\
\text { Median (IQR) }\end{array}$ & $19.4(7.35)$ & $19.5(9.0)$ & & .41 & 0.15 \\
\hline $\begin{array}{l}\text { Pathologic T stage } \\
\text { T1mi } \\
\text { T1a } \\
\text { T1b } \\
\text { T1c }\end{array}$ & $\begin{array}{c}0 \\
8 \\
62 \\
46\end{array}$ & $\begin{array}{l}0 \\
8 \\
62 \\
46\end{array}$ & & 1 & \\
\hline $\begin{array}{l}\text { Dominant subtypes in the invasive component } \\
\text { Papillary } \\
\text { Acinar } \\
\text { Solid } \\
\text { Micropapillary }\end{array}$ & $\begin{array}{c}88 \\
14 \\
12 \\
2\end{array}$ & $\begin{array}{c}46 \\
17 \\
51 \\
2\end{array}$ & & $<.01$ & \\
\hline $\begin{array}{l}\text { Lymphatic invasion* } \\
\text { Absent } \\
\text { Present }\end{array}$ & $\begin{array}{c}110(95) \\
6(5)\end{array}$ & $\begin{array}{c}105(91) \\
11(9)\end{array}$ & $1.18(0.03-54.6)$ & .93 & 0.17 \\
\hline $\begin{array}{l}\text { Vascular invasion* } \\
\text { Absent } \\
\text { Present }\end{array}$ & $\begin{array}{l}91(78) \\
25(22)\end{array}$ & $\begin{array}{l}75(65) \\
41(35)\end{array}$ & $1.52(0.35-6.61)$ & .57 & 0.31 \\
\hline $\begin{array}{l}\text { EGFR mutation } \\
\text { Absent } \\
\text { Present } \\
\text { Not examined }\end{array}$ & $\begin{array}{l}15(13) \\
35(30) \\
66(57)\end{array}$ & $\begin{array}{l}14(12) \\
15(13) \\
87(75)\end{array}$ & & .15 & \\
\hline
\end{tabular}

$C I$, Confidence interval; $C E A$, carcinoembryonic antigen; $I Q R$, interquartile range; $E G F R$, epidermal growth factor receptor. *Included in the logistic regression model.

staining intensity, ranging from 0 to 200 , in each slide. We excluded 3 laminin5-stained slides (2 Lep+ cases; 1 Lep- case) because of unsatisfactory staining. Evaluation of scoring for podoplanin (PDPN) + CAFs was calculated on the basis of the staining intensity and the stained percentage of CAFs with the method described next. CD204+ TAMs in the stroma of the tumor-invasive component were enumerated across 3 high-power fields and averaged.

\section{Survival Analysis}

All patients were basically followed up for 5 years after surgery at least and up to 10 years. Recurrence or survival information was obtained as possible by letters in the case of patients who cannot make regular clinics visit up to 10 years. The length of overall survival was defined as the period between the date of surgery and the last follow-up date or death due to any cause. In the same way, the period of relapse-free survival was calculated in months from the date of resection to the date of first recurrence or last follow-up and death from any cause. Observations were censored at the last follow-up when the patient was alive or lost to follow-up. The data cutoff date was May 2016 at our institution.

\section{Statistical Methods}

We performed matching for patients on the basis of the invasive component size using the following algorithm: 1:1 optimal match with no caliper and no replacement. Associations between the clinicopathologic factors and the presence of lepidic growth were analyzed using the multivariate logistic regression analysis (for categoric variables) or the Mann-Whitney $U$ test (for continuous variables). Histologic subtypes and IHC results were assessed using the Mann-Whitney $U$ test. Overall survival (in months) was calculated from the date of resection to the 
date of death from any cause, and relapse-free survival was calculated to the date of death from any cause and first recurrence or last follow-up. Survival probabilities were calculated by a Kaplan-Meier method and assessed by a Cox regression analysis adjusted for some factors (age, sex, smoking status, total tumor size). Observations were censored at the date of last follow-up when the patient was alive or when the patient was lost to follow-up. The date of data cutoff was May in 2016 at our institution. All data were analyzed with EZR version 1.32, a graphical user interface for R (The R Foundation for Statistical Computing). ${ }^{16}$ More precisely, EZR is a modified version of $\mathrm{R}$ commander that incorporates frequently used biostatistical functions.

\section{RESULTS}

\section{Clinicopathologic Differences Between Lep + and Lep- Adenocarcinomas}

We identified 459 Lep+ and 116 Lep- adenocarcinomas, the clinicopathologic differences between which are shown in Table 1. No significant differences in patient age, sex distribution, preoperative serum carcinoembryonic antigen levels, or total tumor size were detected, but we did observe significant differences in the smoking history of the 2 groups. With respect to pathologic characteristics, Lep + adenocarcinomas exhibited a smaller invasive component size $(10.5 \mathrm{vs} 19.5 \mathrm{~mm}$.; $P<.01)$ and were more likely to be early pathologic $\mathrm{T}$ stage $(P<.01)$. The frequencies of both vascular and lymphovascular invasion were not significantly different between the 2 groups, whereas the frequency of epidermal growth factor receptor mutations was significantly higher in Lep+ adenocarcinomas $(P<.01)$.

\section{Clinicopathologic Differences of Tumors Matched for Invasive Component Size}

Table 2 shows the clinicopathologic features of Lep+ and Lep- tumors matched for invasive component size. There were no significant differences in invasive component size or pathologic T status between the 2 groups. Most of their invasive components were composed of mixed subtypes, but the dominant subtypes in the invasive component were significantly different between the 2 groups $(P<.01)$. Almost all of the differences of characteristics were not significant, but total tumor size was significantly greater in Lep+ adenocarcinomas ( 25 vs $20 \mathrm{~mm}, P<.01)$.

\section{Prognostic Impact of the Presence of Lepidic Growth}

Figure E1 shows the Kaplan-Meier curves for the overall and relapse-free survival of patients with Lep+ and Lepadenocarcinoma. We did not observe significant differences for both overall survival and relapse-free survival between the 2 groups. There were also no significant survival differences between Lep+ and Lep- adenocarcinomas, which were performed by using IHC staining (Figure E2).

\section{Immunohistochemical Staining Score}

The IHC staining results are summarized in Tables 3 and 4 .
TABLE 3. Immunohistochemical staining score of cancer cells

\begin{tabular}{|c|c|c|c|}
\hline & Lepidic (+) & Lepidic (-) & $\boldsymbol{P}^{*}$ \\
\hline \multicolumn{4}{|l|}{ CD44 } \\
\hline Median (IQR) & $50(50)$ & $20(50)$ & $<.01$ \\
\hline \multicolumn{4}{|l|}{ ALDH1 } \\
\hline Median (IQR) & $40(70)$ & $10(70)$ & .33 \\
\hline \multicolumn{4}{|l|}{ GLUT-1 } \\
\hline Median (IQR) & $0(10)$ & $10(20)$ & $<.01$ \\
\hline \multicolumn{4}{|l|}{ CA IX } \\
\hline Median (IQR) & $0(0)$ & $0(20)$ & .01 \\
\hline \multicolumn{4}{|l|}{ Laminin-5 } \\
\hline Median (IQR) & $0(10)$ & $0(10)$ & .10 \\
\hline
\end{tabular}

1. Evaluation of cancer cells

(1) Cancer stem cell-related molecule

Cancer cell-specific ALDH1, which exhibited a cytoplasmic staining pattern, did not differ between the 2 groups. As for the staining of CD44, expressed in the membrane and cytoplasm of cancer cell, the score was significantly higher in Lep+ adenocarcinomas $(50$ vs $20, P<.01)$ (Figure 2).

(2) Hypoxia-related molecules

Lep + adenocarcinomas exhibited significantly lower GLUT1 and CA IX staining scores (GLUT1: 0 vs $10, P<.01$; CA IX: 0 vs 0 [mean: 4.7 vs 14.1 ], $P=.01$ ) (Figure 2 ).

(3) Invasion-related molecule

There were no significant differences in the expression levels of laminin- 5 between the 2 groups.

2. Evaluation of stromal cells

(1) $\mathrm{PDPN}+\mathrm{CAFs}$

The staining score for PDPN+ CAFs was significantly lower in Lep+ adenocarcinomas (0 vs 0 (mean: 1.6 vs 11.6$), P<.01$ ) (Figure 2 ).

(2) M2 macrophages

The number of CD204+ TAMs was significantly lower in Lep+ adenocarcinomas (8.7 vs 24.7, $P<.01$ ) (Figure 2).

TABLE 4. Immunohistochemical staining score of stromal cells

\begin{tabular}{lccc}
\hline & Lepidic (+) & Lepidic (-) & $\boldsymbol{P}^{*}$ \\
\hline $\begin{array}{c}\text { PDPN } \\
\text { Median }(\mathrm{IQR})\end{array}$ & $0(0)$ & $0(10)$ & $<.01$ \\
$\begin{array}{c}\text { CD204 } \\
\text { Median (IQR) }\end{array}$ & $8.7(15.3)$ & $24.7(27.3)$ & $<.01$ \\
\hline
\end{tabular}

$P D P N$, Podoplanin; $C A F$, cancer-associated fibroblast; $I Q R$, interquartile range; TAM, tumor-associated macrophage. *Calculated by Mann-Whitney $U$ test. 
lepidic positive group

CD44

GLUT-1
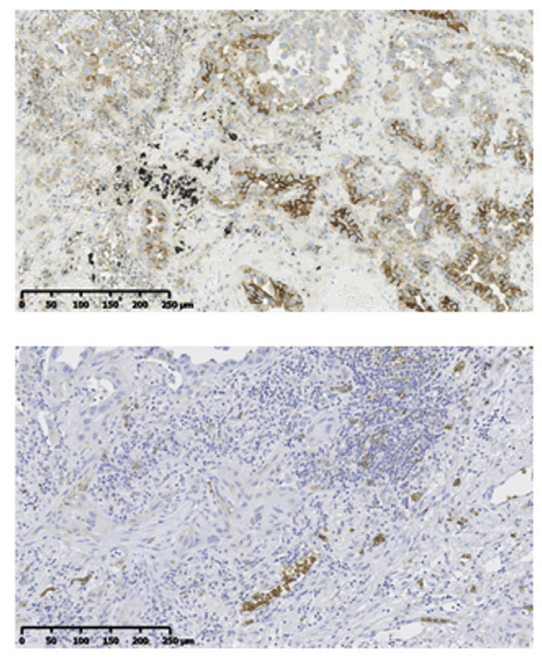

CA IX

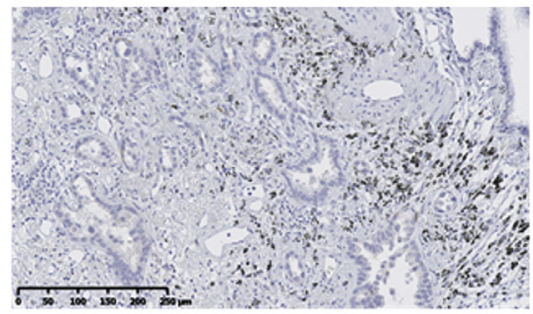

PDPN

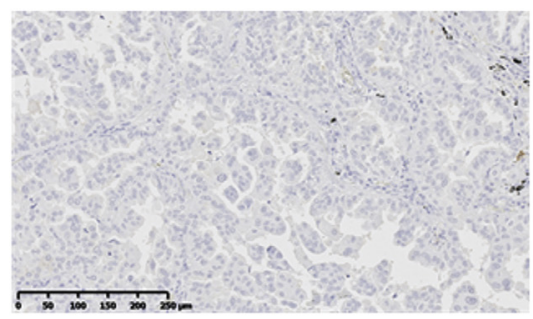

CD204

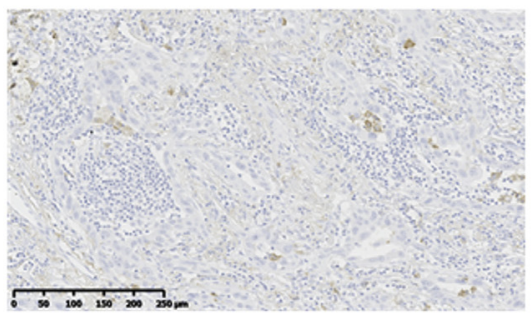

Laminin-5

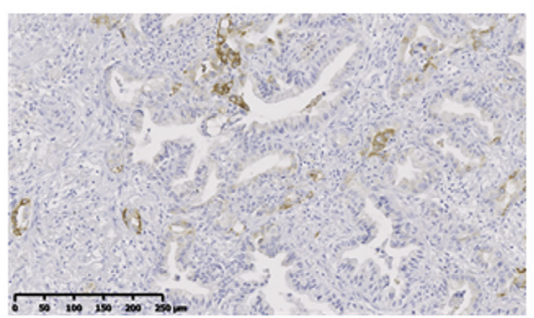

lepidic negative group
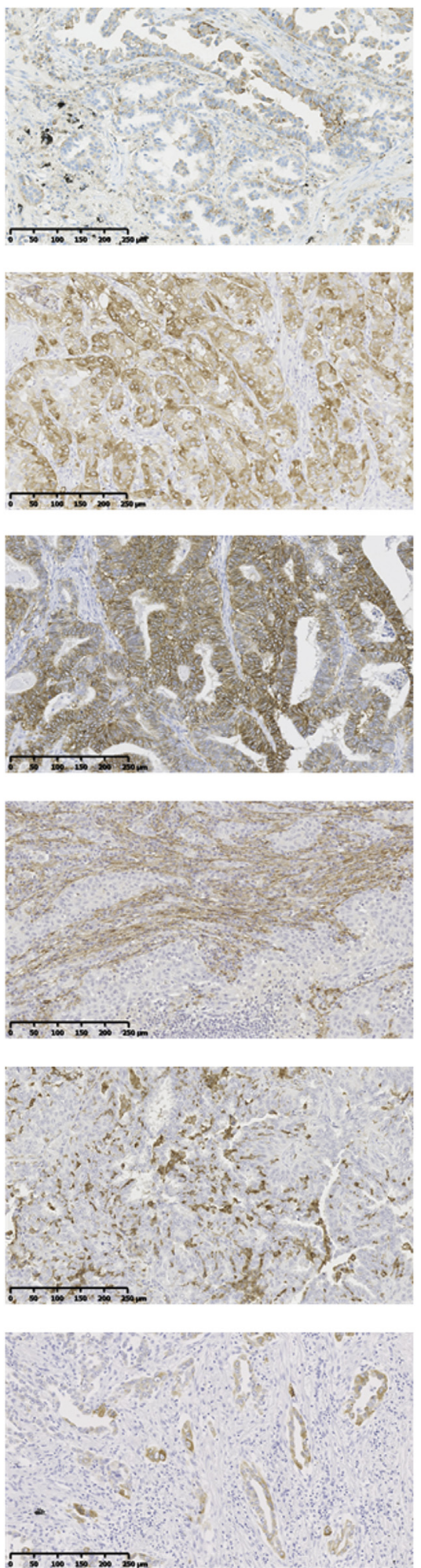

FIGURE 2. Distinct IHC staining patterns of Lep+ and Lep- adenocarcinomas. Left: Lep+ adenocarcinomas; the staining score of CD44 is higher. Right: Lep- adenocarcinomas; the staining scores of hypoxic marker (GLUT-1, CA IX) and tumor-promoting stromal cells (PDPN + CAFs and CD204 + TAMs) are higher. There were no significant differences in the expression levels of laminin- 5 between the 2 groups. 


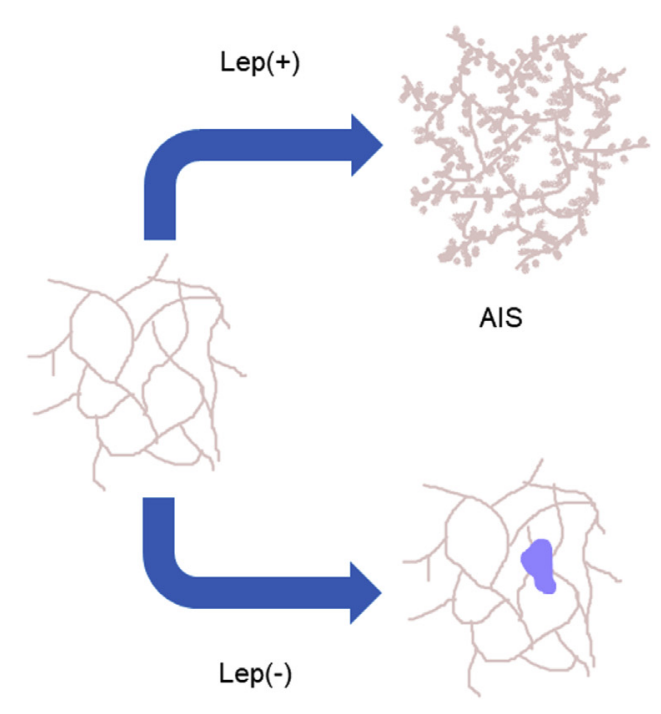

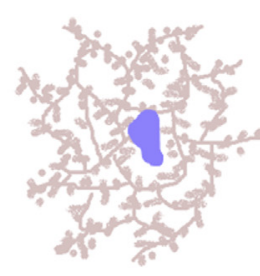

MIA

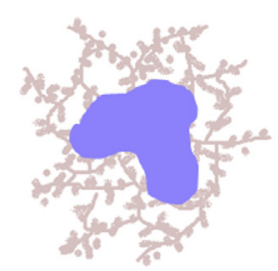

LPA

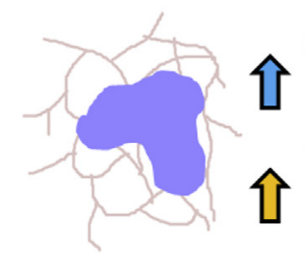

Hypoxic marker

GLUT-1

CAIX

Tumor promoting stromal cells

CD204+TAMs

PDPN $^{+}$CAFs

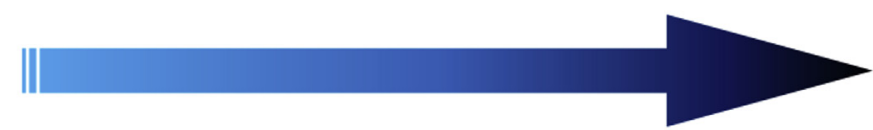

FIGURE 3. Schematic of a model for Lep + and Lep- adenocarcinoma development. Upper: Lep + adenocarcinomas progress slowly with preservation of the alveolar structure as stepwise progression, which can be maintained with co-opted vessels. Lower: Lep- adenocarcinomas often grow rapidly and destructively, and widespread vascular disruption and regression of co-opted vessels may occur in the invasive area, resulting in a hypoxic core. AIS, Adenocarcinoma in situ; MIA, minimally invasive adenocarcinoma; $L P A$, lepidic predominant adenocarcinoma.

\section{DISCUSSION}

In this study, we compared the clinicopathologic features of Lep + and Lep- adenocarcinomas with size-matched invasive components. In survival analysis, there were no significant differences between Lep + and Lep- adenocarcinomas. On IHC staining (Figure 3), both hypoxia marker scores (GLUT-1 and CA IX) and scores of the tumor-promoting stromal cells (CD204+ TAMs and $\mathrm{PDPN}+\mathrm{CAFs}$ ) were significantly lower in Lep + adenocarcinomas. These results indicate that the invasive component of Lep+, but not Lep-, adenocarcinoma is associated with a less malignant microenvironment. To our knowledge, this is the first study to clarify the clinicopathologic differences between the invasive components of Lep+ and Lep- adenocarcinomas.

GLUT-1 and CA IX are established hypoxia markers. Hypoxia is an important factor that influences tumor proliferation and malignant progression. ${ }^{14,15}$ Elevated expression of GLUT-1 and CA IX in solid cancers, including lung cancer, is known as a poor prognostic factor and is associated with a greater risk of vascular invasion and metastasis. ${ }^{17-21}$ In our study, the expression of GLUT-1 and CA IX was significantly attenuated in Lep + adenocarcinomas. This result indicates that the invasive component of Lep + adenocarcinoma exhibits a reduced invasive potential. $\operatorname{Lep}(+)$ adenocarcinomas had a significantly lower percentage of solid component than
Lep(-) adenocarcinomas (Table E2). Chiu and colleagues ${ }^{22}$ reported the strong correlation of solid growth pattern and GLUT1 expression, and this result was consistent with ours.

Compared with Lep- adenocarcinomas, Lep+ tumors contained a lower number of PDPN $+\mathrm{CAFs}$ and CD204+ TAMs (M2 macrophages). Given that these stromal cells promote cancer cell invasion and tumor metastasis, ${ }^{23-25}$ we conclude that Lep+ adenocarcinomas are associated with a less-invasive microenvironment.

By using an animal hypoxia model, Zhang and colleagues $^{26}$ reported that hypoxia significantly promotes lung cancer metastasis accompanied by an increased infiltration of M2 macrophages. In addition, Kolenda and colleagues $^{27}$ reported that with human glioma cell lines LN308 and U87MG cells, a robust increase in PDPN mRNA was observed with those in the condition of 72hour hypoxia compared with those in the normoxic condition. Therefore, lower numbers of tumor-promoting stromal cells in Lep+ adenocarcinomas may be associated with less hypoxic conditions.

In the present study, expression levels of CD44 were significantly higher in Lep + adenocarcinomas. CD44 expression is reportedly correlated with long survival and predominantly lepidic subtypes in lung adenocarcinoma, consistent, in part, with our results. ${ }^{28}$ In fact, one of the supportive explanations is that the interaction between CD44 
and its ligand of hyaluronic acid may inhibit angiogenesis and progression of cancers. ${ }^{29}$

The current World Health Organization classification scheme proposes that the new adenocarcinoma in situ and minimally invasive carcinoma categories represent a stepwise progression of Lep+ adenocarcinoma. Naito and colleagues $^{11}$ reported that this morphologic progression is associated with elevated cancer cell-specific expression of invasion-related molecules, as well as increased recruitment of tumor-promoting stromal cells. Thus, Lep + adenocarcinomas may slowly acquire malignant potential as their invasive components undergo stepwise changes (ie, "multistage carcinogenesis") (Figure 3).

On the contrary, the mechanism of Lep- adenocarcinoma tumorigenesis is poorly understood. In a process known as vessel (or vascular) co-option, tumor cells may grow adjacent to preexisting blood vessels, thereby facilitating tumor cell-mediated acquisition of oxygen and essential nutrients without obligate neovascularization. ${ }^{30-32}$ Progression of Lep+ adenocarcinoma can be supplied with co-opted vessels, because they grow up slowly with preservation of the alveolar structure. However, Lep- adenocarcinoma often grows rapidly and destructively, leading to widespread disruption of coopted vessels and the development of a hypoxic core (Figure 3). ${ }^{32,33}$

A previous study reported a strong correlation between the sizes of solid and invasive tumor components as determined by thin-section CT. ${ }^{34}$ So, given that tumors lacking GGO on thin-section CT are likely Lep- adenocarcinomas, treatment strategies for those tumors, especially performing limited resection, must be carefully selected, and additional preoperative examination such as positron emission tomography CT may provide more information. ${ }^{35}$

We investigated patients who underwent lobectomy, and the survival differences between the 2 groups were slight but not significant. However, the opportunity of performing limited resection for the patients with small-size tumors has been increasing, and our IHC results suggest that performing limited resection for Lep(-) adenocarcinomas may have the possibility of undertreatment. Therefore, careful patient selection will be required when we plan on performing limited resection. To clarify this hypothesis, further investigation for survival data from patients with Lep(-) adenocarcinoma who receive sublobar resection is necessary.

\section{Study Limitations}

First, this study was retrospective and conducted in a single institution. Second, in the IHC staining scoring, we could not be blinded to the presence or absence of lepidic component. Third, the associations we observed may be due to chance, so further verification in other studies will be required.

\section{CONCLUSIONS}

The current study indicated that lower levels of cancer cell-specific hypoxia marker expression and a smaller number of tumor-promoting stromal cells in the invasive component were characteristic features of Lep + adenocarcinoma. These results suggest that the invasive component of Lep + adenocarcinoma is associated with a less malignant tumor microenvironment. Given that Lep + adenocarcinoma is less invasive with less metastatic potential, early-stage small Lep+ adenocarcinomas may be treated with limited resection techniques such as wide-wedge resection and segmentectomy. Further investigation based on survival data from patients with Lep(-) adenocarcinoma who receive sublobar resection will be required.

\section{Conflict of Interest Statement}

Dr Tsuboi reports personal fees from AstraZeneca KK, Eli Lily Japan, Boehringer-Ingelheim Japan, and Daiichi-Sankyo, all outside the conduct of the study. Dr Kuwata reports personal fees from Chugai Pharma, Daiichi-Sankyo, and Astra-Zeneca KK. All other authors have nothing to disclose with regard to commercial support.

\section{References}

1. Siegel RL, Miller KD, Jemal A. Cancer statistics, 2016. CA Cancer J Clin. 2016; 66:7-30.

2. Travis WD, Asamura H, Bankier AA, Beasley MB, Detterbeck F, Flieder DB, et al. The IASLC Lung Cancer Staging Project: Proposals for Coding T Categories for Subsolid Nodules and Assessment of Tumor Size in Part-Solid Tumors in the Forthcoming Eighth Edition of the TNM Classification of Lung Cancer. $J$ Thorac Oncol. 2016;11:1204-23.

3. Kadota K, Villena-Vargas J, Yoshizawa A, Motoi N, Sima CS, Riely GJ, et al. Prognostic significance of adenocarcinoma in situ, minimally invasive adenocarcinoma, and nonmucinous lepidic predominant invasive adenocarcinoma of the lung in patients with stage I disease. Am J Surg Pathol. 2014;38:448-60.

4. Yoshizawa A, Motoi N, Riely GJ, Sima CS, Gerald WL, Kris MG, et al. Impact of proposed IASLC/ATS/ERS classification of lung adenocarcinoma: prognostic subgroups and implications for further revision of staging based on analysis of 514 stage I cases. Mod Pathol. 2011;24:653-64.

5. Asamura H, Goya T, Koshiishi Y, Sohara Y, Eguchi K, Mori M, et al. A Japanese Lung Cancer Registry study: prognosis of 13,010 resected lung cancers. J Thorac Oncol. 2008;3:46-52.

6. Terasaki H, Niki T, Matsuno Y, Yamada T, Maeshima A, Asamura H, et al. Lung adenocarcinoma with mixed bronchioloalveolar and invasive components: clinicopathological features, subclassification by extent of invasive foci, and immunohistochemical characterization. Am J Surg Pathol. 2003;27:937-51.

7. Sakurai H, Maeshima A, Watanabe S, Suzuki K, Tsuchiya R, Maeshima AM, et al. Grade of stromal invasion in small adenocarcinoma of the lung: histopathological minimal invasion and prognosis. Am J Surg Pathol. 2004;28:198-206.

8. Noguchi M, Shimosato Y. The development and progression of adenocarcinoma of the lung. In: Hansen HH, ed. Lung Cancer: Advances in Basic and Clinical Research. Boston, MA: Springer US; 1995:131-42.

9. Ishii G, Ochiai A, Neri S. Phenotypic and functional heterogeneity of cancerassociated fibroblast within the tumor microenvironment. Adv Drug Deliv Rev. 2016;99:186-96.

10. Ito M, Ishii G, Nagai K, Maeda R, Nakano Y, Ochiai A. Prognostic impact of cancer-associated stromal cells in patients with stage I lung adenocarcinoma. Chest. 2012;142:151-8.

11. Naito M, Aokage K, Saruwatari K, Hisakane K, Miyoshi T, Hishida T, et al Microenvironmental changes in the progression from adenocarcinoma in situ to minimally invasive adenocarcinoma and invasive lepidic predominant adenocarcinoma of the lung. Lung Cancer. 2016;100:53-62. 
12. Hattori A, Matsunaga T, Takamochi K, Oh S, Suzuki K. Prognostic impact of a ground glass opacity component in the clinical T classification of non-small cell lung cancer. J Thorac Cardiovasc Surg. 2017;154:2102-10.e2101.

13. Moon Y, Sung SW, Lee KY, Kim YK, Park JK. The importance of the lepidic component as a prognostic factor in stage I pulmonary adenocarcinoma. World J Surg Oncol. 2016;14:37.

14. Thiery JP. Epithelial-mesenchymal transitions in tumour progression. Nature Rev Cancer. 2002;2:442-54.

15. Onder TT, Gupta PB, Mani SA, Yang J, Lander ES, Weinberg RA. Loss of E-cadherin promotes metastasis via multiple downstream transcriptional pathways. Cancer Res. 2008;68:3645-54.

16. Kanda Y. Investigation of the freely available easy-to-use software 'EZR' for medical statistics. Bone Marrow Transplant. 2013;48:452-8.

17. Pardridge WM, Boado RJ, Farrell CR. Brain-type glucose transporter (GLUT-1) is selectively localized to the blood-brain barrier. Studies with quantitative western blotting and in situ hybridization. J Biol Chem. 1990;265:18035-40.

18. Osugi J, Yamaura T, Muto S, Okabe N, Matsumura Y, Hoshino M, et al. Prognostic impact of the combination of glucose transporter 1 and ATP citrate lyase in nodenegative patients with non-small lung cancer. Lung Cancer. 2015;88:310-8.

19. Wang J, Ye C, Chen C, Xiong H, Xie B, Zhou J, et al. Glucose transporter GLUT1 expression and clinical outcome in solid tumors: a systematic review and metaanalysis. Oncotarget. 2017;8:16875-86.

20. Sawayama H, Ishimoto T, Watanabe M, Yoshida N, Baba Y, Sugihara H, et al. High expression of glucose transporter 1 on primary lesions of esophageal squamous cell carcinoma is associated with hematogenous recurrence. Ann Surg Oncol. 2014;21:1756-62.

21. van Kuijk SJ, Yaromina A, Houben R, Niemans R, Lambin P, Dubois LJ. Prognostic significance of carbonic anhydrase IX expression in cancer patients: a meta-analysis. Front Oncol. 2016;6:69.

22. Chiu C-H, Yeh Y-C, Lin K-H, Wu Y-C, Lee Y-C, Chou T-Y, et al. Histological subtypes of lung adenocarcinoma have differential ${ }^{18} \mathrm{~F}$-fluorodeoxyglucose uptakes on the positron emission tomography/computed tomography scan. J Thorac Oncol. 2011;6:1697-703.

23. Ohtaki Y, Ishii G, Nagai K, Ashimine S, Kuwata T, Hishida T, et al. Stromal macrophage expressing CD204 is associated with tumor aggressiveness in lung adenocarcinoma. J Thorac Oncol. 2010;5:1507-15.

24. Hoshino A, Ishii G, Ito T, Aoyagi K, Ohtaki Y, Nagai K, et al. Podoplanin-positive fibroblasts enhance lung adenocarcinoma tumor formation: podoplanin in fibroblast functions for tumor progression. Cancer Res. 2011;71:4769-79.
25. Neri S, Ishii G, Hashimoto H, Kuwata T, Nagai K, Date H, et al. Podoplanin-expressing cancer-associated fibroblasts lead and enhance the local invasion of cancer cells in lung adenocarcinoma. Int J Cancer. 2015;137:784-96.

26. Zhang J, Cao J, Ma S, Dong R, Meng W, Ying M, et al. Tumor hypoxia enhances non-small cell lung cancer metastasis by selectively promoting macrophage M2 polarization through the activation of ERK signaling. Oncotarget. 2014;5:9664-77.

27. Kolenda J, Jensen SS, Aaberg-Jessen C, Christensen K, Andersen C, Brunner N, et al. Effects of hypoxia on expression of a panel of stem cell and chemoresistance markers in glioblastoma-derived spheroids. J Neuro-oncol. 2011;103: 43-58.

28. Park E, Park SY, Sun PL, Jin Y, Kim JE, Jheon S, et al. Prognostic significance of stem cell-related marker expression and its correlation with histologic subtypes in lung adenocarcinoma. Oncotarget. 2016;7:42502-12.

29. Toole BP, Slomiany MG. Hyaluronan: a constitutive regulator of chemoresistance and malignancy in cancer cells. Semin Cancer Biol. 2008;18:244-50.

30. Bergers G, Benjamin LE. Tumorigenesis and the angiogenic switch. Nat Rev Cancer. 2003;3:401-10.

31. Folkman J. Tumor angiogenesis: therapeutic implications. N Engl J Med. 1971; 285:1182-6.

32. Holash J, Wiegand SJ, Yancopoulos GD. New model of tumor angiogenesis: dynamic balance between vessel regression and growth mediated by angiopoietins and VEGF. Oncogene. 1999;18:5356-62.

33. Korn C, Augustin HG. Mechanisms of vessel pruning and regression. Dev Cell. 2015;34:5-17.

34. Aokage K, Miyoshi T, Ishii G, Kusumoto M, Nomura S, Katsumata S, et al. Clinical and pathological staging validation in the Eighth Edition of the TNM Classification for Lung Cancer: correlation between solid size on thin-section computed tomography and invasive size in pathological findings in the new T classification. J Thorac Oncol. 2017;12:1403-12.

35. Hattori A, Matsunaga T, Takamochi K, Oh S, Suzuki K. Indications for sublobar resection of clinical stage IA radiologic pure-solid lung adenocarcinoma. $J$ Thorac Cardiovasc Surg. 2017;154:1100-8.

Key Words: lung adenocarcinoma, lepidic, invasive component, tumor microenvironment, hypoxia, tumor-promoting stromal cells 

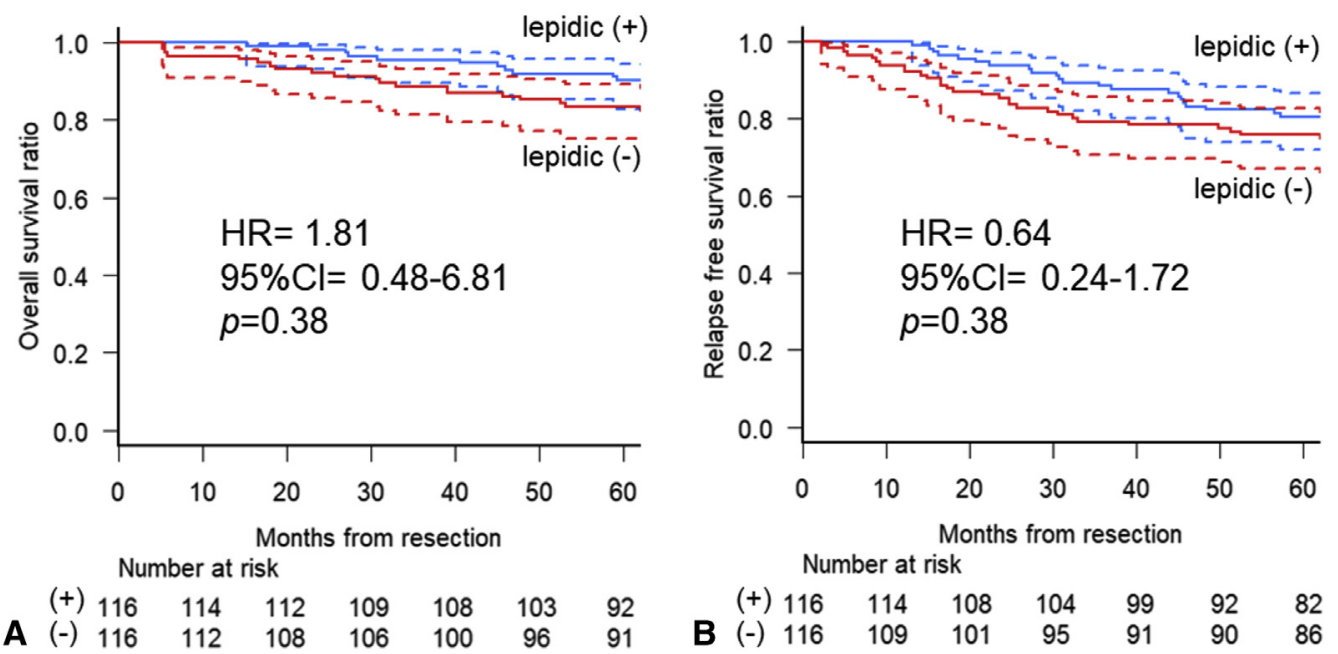

FIGURE E1. Comparison of overall and relapse-free survival between patients with Lep + and Lep- adenocarcinomas (adjusted for age, sex, smoking status, and total tumor size). A, Overall survival. B, Relapse-free survival. (+): Lep+ adenocarcinomas; (-): Lep- adenocarcinomas. HR, Hazard ratio; $C I$, confidence interval.
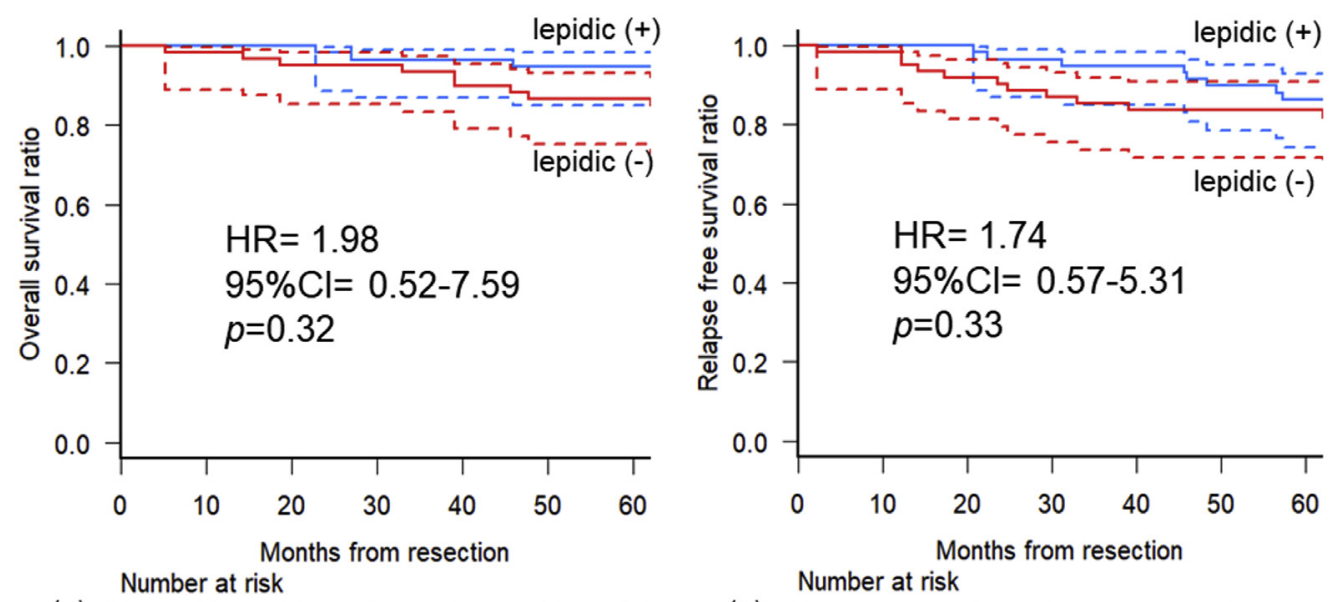

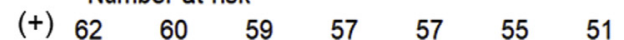

A (-) $\begin{array}{lllllll}61 & 60 & 58 & 58 & 54 & 52 & 50\end{array}$

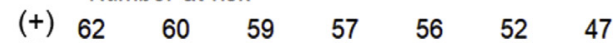

FIGURE E2. Comparison of overall and relapse-free survival between patients with Lep + and Lep- adenocarcinomas, which were performed by using IHC staining (adjusted for age, sex, smoking status, and total tumor size). A, Overall survival. B, Relapse-free survival. $(+)$ : Lep + adenocarcinomas; (-): Lep- adenocarcinomas. HR, Hazard ratio; $C I$, confidence interval. 
TABLE E1. Antibodies used in this study

\begin{tabular}{lccll}
\hline \multicolumn{1}{c}{ Category } & Antibody & Clone & Preparation & \multicolumn{1}{c}{ Source } \\
\hline Stem cell & ALDH-1 & 44/ALDH & Microwave & BD Transduction Laboratory (San Jose, Calif) \\
& CD44 & DF1485 & Microwave & Dako Cytomation (Carpinteria, Calif) \\
Hypoxia & GLUT-1 & N/A & Microwave & Spring Bioscience (Pleasanton, Calif) \\
& CA IX & - & Microwave & Proteintech (Rosemont, Ill) \\
Cell invasion & Laminin-5 & D4B5 & Proteinase K & Millipore (Burlington, Mass) \\
M2 macrophage & CD204 & SRA-E5 & Microwave & TransGenic Inc (Tokyo, Japan) \\
CAFs & PDPN & D2-40 & Microwave & Acris Antibodies, Inc (Rockville, Md) \\
\hline
\end{tabular}

$C A F$, Cancer-associated fibroblast; $P D P N$, podoplanin.

TABLE E2. Difference of the percentage of histologic subtypes comprising invasive component between $\operatorname{Lep}(+)$ and $\operatorname{Lep}(-)$ adenocarcinomas (after matching for invasive component size)

\begin{tabular}{lccc}
\hline & Lepidic (+) & Lepidic (-) & $\boldsymbol{P}^{*}$ \\
\hline $\begin{array}{l}\text { Papillary } \\
\quad \text { Median, \% (IQR) }\end{array}$ & $73.2(48.7)$ & $21.1(80.0)$ & $<.01$ \\
$\begin{array}{l}\text { Acinar } \\
\quad \text { Median, \% (IQR) }\end{array}$ & $15.5(33.3)$ & $10.0(30.0)$ & .90 \\
$\begin{array}{l}\text { Solid } \\
\quad \text { Median, \% (IQR) }\end{array}$ & $0.0(15.6)$ & $20.0(73.7)$ & $<.01$ \\
$\begin{array}{l}\text { Micropapillary } \\
\text { Median, \% (IQR) }\end{array}$ & $0.0(0.0)$ & $0.0(0.0)$ & .55 \\
\hline $\begin{array}{l}\text { IOR, Interquartile range. *Calculated by Mann-Whitney } U \text { test. } \\
\quad\end{array}$
\end{tabular}

$I Q R$, Interquartile range. *Calculated by Mann-Whitney $U$ test. 